B

J. bio-sci. 21: 51-59, 2013

ISSN 1023-8654

http://www.banglajol.info/index.php/JBS/index

\title{
GROWTH ATTRIBUTES OF RAPESEED VARIETIES (BRASSICA CAMPESTRIS L.) IN RELATION TO SOWING DATES AND WEEDINGS TREATMENTS
}

\author{
Mst. Towhida Akhter $\epsilon^{*}$, P B Kundu, N K Paul \\ $€$ Institute of Biological Sciences, University of Rajshahi, Rajshahi-6205, Bangladesh \\ Department of Botany, University of Rajshahi, Rajshahi-6205, Bangladesh
}

\begin{abstract}
Context: To analyze the effect of sowing dates and weeding treatments on growth attributes such as total dry matter (TDM), leaf area index (LAI), net assimilation rate (NAR), crop growth rate (CGR), leaf area ratio (LAR) of three rapeseed (Brassica campestris L.).

Objectives: The objective of the study was to identify the growth in relation to sowing dates and weed competition and identify the weeding number for better growth of rapeseed.

Materials and Methods: This investigation was arranged as factorial experiment based on the split-split plot design with three replications. Four sowing dates were including $18 \operatorname{October}\left(\mathrm{S}_{1}\right), 12 \operatorname{November}\left(\mathrm{S}_{2}\right)$, 17 November $\left(S_{3}\right)$ and 3 December $\left(S_{4}\right)$ and three weeding treatments were no weeding $\left(W_{0}\right)$, one hand weeding $\left(\mathrm{W}_{1}\right)$ and two hand weeding $\left(\mathrm{W}_{2}\right)$ and three varieties viz., BINA Sarisha-5, BINA Sarisha- 6 and BARI Sarisha-14 were investigated.

Results: The results showed that, TDM, LAI, CGR, NAR and LAR were affected by sowing date and weeding treatments. The highest TDM, LAI and CGR were obtained in the first sowing and two weedings treatment. The highest NAR was found in $S_{2}$ plants and $W_{0}$ treatment. The highest LAR was found in $S_{2}$ plants and $W_{1}$ treatment.

Conclusion: The results obtained in this study suggest that the first sowing and two weeding treatments are superior for the production of rapeseed.
\end{abstract}

Key words: Rapeseed, Sowing date, weeding, growth attributes.

Introduction

Rapeseed (Brassica campestris L.) ranks first among the oilseed crops of Bangladesh. Rapeseed occupies almost $71 \%$ land whereas the other oilseeds in the rest of the land. It covers about $61.2 \%$ of the total acreage under oilseed and $58 \%$ of the total oil seed production (BBS. 2010). Rapeseed has a better teste as well as highest level of unsaturated fatty acid. And thus helps lowering blood cholesterol levels (Marwat et al. 2003). It is also a rich source of oil and protein and contains more than $40 \%$ oil (Weiss1983).

As wheat and rice are the staple food crop of Bangladesh, so lesser attention is given to oilseed crops of rabi season. That is why rapeseed is only grown on almost rainfed and less fertile areas. As a result not enough edible oil is produced to fulfil the domestic requirement. So edible oil is imported by spending a lot of foreign exchange. Many physiological processes associated with crop growth and developments are influenced by sowing date. Appropriate time of sowing enables the crop plant to enjoy suitable climatic conditions for their growth and development which largely influence yield (Paul and Sarker 2003).

Rapeseed is a smoother crop because of its larger leaves, rapid growth and early canopy closing. Still weed competition is very critical during the early stand establishment particularly the parasitic weed (Joel et al. 1995). Due to smaller seed size of the crop it is difficult to separate weed seeds from it. Several methods

\footnotetext{
* Corresponding address E-mail: towhida_akhter@yahoo.com
} 
have been used for weed control in canola, like hand weeding, cultivation in row cropping and use of chemicals. But most reliance is made on hand weeding. Khan et al. (1995) suggested the use of postemergence with hand weeding for controlling weeds in rapeseed if proper pre-emergence herbicide is not available. The present study was undertaken to investigate the impact of optimum sowing date and suitable weeding treatment on growth dynamics of three rapeseed varieties.

\section{Materials and Methods}

\section{Experimental field}

The experiment was carried out at the experimental field of Rajshahi University Campus (Agro-Ecological Zone 11), Bangladesh (2475 N latitude and $9050 \mathrm{E}$ longitude) during the period from October 2006 to March 2007 and October 2008 to March 2009 growing seasons. The soil type was silty loam, having pH 7.5 as well as $35 \%$ of field capacity.

\section{Treatment}

The experiment was laid out in a split-split plot design with three replications. Each replicated field was divided into four main plots for sowing treatments $\left(S_{1}=18\right.$ October, $S_{2}=2$ November, $S_{3}=17$ November, $S_{4}$ $=3$ December). Each main plot was divided into three sub-plots for weeding treatment $\left(W_{0}=\right.$ no weeding, $W_{1}$ $=$ one hand weeding, $W_{2}=$ two hand weedings). Each sub-plot was lastly divided into four sub-sub plots for three varieties (namely BINA Sarisha-5, BINA Sarisha-6 and BARI Sarisha-14 ) of rapeseed. Each plot size was $4 \mathrm{~m} 3 \mathrm{~m}$, i.e., $12 \mathrm{~m}^{2}$ having a plot to plot distance $1 \mathrm{~m}$ to the North-South, $2 \mathrm{~m}$ to the East-West; replication to replication distance was $2 \mathrm{~m}$, row to row $30 \mathrm{~cm}$, and plant to plant $10 \mathrm{~cm}$ approximately. Border rows were not considered because of the border effect.

\section{Data analysis}

At harvest, the three middle rows were used for sampling. For growth analysis, ten plants from the middle of each plot were harvested at 7 days intervals and the first harvest was taken at 20 days after sowing (DAS).The following traits were studied; TDM, LAI, CGR, NAR and LAR. Analysis of variance was performed with a personal computer using the IRRISTAT software. A factorial analysis of variance (ANOVA) was performed for all the parameters. Statistical analysis was carried out according to Gomez and Gomez (1984).

\section{Results and Discussion}

Analysis of variance indicated that the differences among the sowing dates and weeding treatments were found to be significant for all the growth attributes at most of the growth stages.

\section{Effect of sowing date}

In the present experiment, the highest TDM was found to be in plants grown under $S_{1}$ condition and lowest under $S_{4}$ condition at all the stages of growth (Table 1). Similar results were reported in rapeseed by Dehdeshti et al. (2006) and Hokmalipour et al. (2011). TDM increased slowly at the early stages of growth and then increased rapidly with the advancement of plant age. Similar result was reported in rapeseed by Kundu and Paul (1998), Dehdeshti et al. (2006) and Hokmalipour et al. (2011). S1 plants had higher LAl than the plants of other sowings (Table 2). Similar result was reported by Clarke and Simpson (1987) and Paul and Kundn (1991) in rape and Hussain et al. (1997) and Ghosh et al. (2003) in mustard. Starting from lower value, LAI reached a certain value and then declined with plant age. Similar result was reported by Prodhan and Ghosh (1986) and Samanta et al. (1997). 
Table 1. Mean values of total dry matter $\left(\mathrm{gm}^{-2}\right)$ at different growth stages as influenced by sowing date (mean of three replications) in two seasons.

\begin{tabular}{|c|c|c|c|c|c|c|c|c|}
\hline \multirow{2}{*}{ Sowing } & \multicolumn{8}{|c|}{ Days after sowing } \\
\hline & 21 & 28 & 35 & 42 & 49 & 56 & 63 & 70 \\
\hline \multicolumn{9}{|c|}{ 2006-2007 } \\
\hline $\mathrm{S}_{1}(18$ October) & 5.791 & 18.647 & 62.420 & 153.484 & 205.261 & 258.718 & 328.468 & 352.207 \\
\hline $\mathrm{S}_{2}$ (02 November) & 5.620 & 16.464 & 39.000 & 83.145 & 104.584 & 156.243 & 232.179 & 254.845 \\
\hline $\mathrm{S}_{3}$ (17 November) & 3.425 & 11.635 & 25.335 & 47.916 & 85.680 & 109.328 & 156.567 & 162.215 \\
\hline $\mathrm{S}_{4}(03$ December $)$ & 2.449 & 9.557 & 18.348 & 32.733 & 65.499 & 86.231 & 112.999 & 117.100 \\
\hline LSD(5\%) & 0.33 & 2.72 & 4.63 & 3.64 & 7.70 & 7.35 & 11.70 & 10.57 \\
\hline \multicolumn{9}{|c|}{ 2008-2009 } \\
\hline $\mathrm{S}_{1}(18$ October) & 4.249 & 14.127 & 41.795 & 116.191 & 190.088 & 241.056 & 311.223 & 362.695 \\
\hline $\mathrm{S}_{2}$ (02 November) & 4.223 & 11.429 & 33.040 & 75.113 & 103.911 & 144.064 & 211.067 & 230.193 \\
\hline $\mathrm{S}_{3}$ (17 November) & 3.113 & 9.928 & 23.347 & 39.444 & 81.393 & 103.756 & 145.680 & 151.072 \\
\hline $\mathrm{S}_{4}(03$ December) & 2.251 & 7.837 & 17.408 & 30.763 & 62.425 & 83.803 & 105.212 & 106.617 \\
\hline LSD(5\%) & 0.19 & 0.62 & 3.38 & 3.63 & 2.62 & 6.40 & 5.33 & 8.05 \\
\hline
\end{tabular}

Table 2. Mean values of leaf area index at different growth stages as influenced by sowing date (mean of three replications) in two seasons.

\begin{tabular}{|c|c|c|c|c|c|c|c|c|}
\hline \multirow{2}{*}{ Sowing } & \multicolumn{8}{|c|}{ Days after sowing } \\
\hline & 21 & 28 & 35 & 42 & 49 & 56 & 63 & 70 \\
\hline & \multicolumn{8}{|c|}{$2006-2007$} \\
\hline $\mathrm{S}_{1}(18$ October) & 0.119 & 0.392 & 0.974 & 1.410 & 0.879 & 0.557 & 0.294 & 0.159 \\
\hline $\mathrm{S}_{2}(02$ November) & 0.135 & 0.365 & 0.632 & 0.837 & 0.413 & 0.314 & 0.234 & 0.146 \\
\hline $\mathrm{S}_{3}(17$ November $)$ & 0.070 & 0.231 & 0.432 & 0.531 & 0.396 & 0.304 & 0.222 & 0.098 \\
\hline $\mathrm{S}_{4}(03$ December $)$ & 0.052 & 0.181 & 0.316 & 0.378 & 0.386 & 0.304 & 0.209 & 0.080 \\
\hline \multirow[t]{2}{*}{$\operatorname{LSD}(5 \%)$} & 0.025 & 0.058 & 0.102 & 0.198 & 0.059 & 0.076 & 0.038 & 0.021 \\
\hline & \multicolumn{8}{|c|}{ 2008-2009 } \\
\hline $\mathrm{S}_{1}(18$ October) & 0.085 & 0.287 & 0.745 & 1.145 & 0.838 & 0.541 & 0.289 & 0.119 \\
\hline $\mathrm{S}_{2}(02$ November) & 0.092 & 0.262 & 0.541 & 0.785 & 0.392 & 0.383 & 0.221 & 0.099 \\
\hline $\mathrm{S}_{3}(17$ November $)$ & 0.064 & 0.218 & 0.353 & 0.381 & 0.358 & 0.317 & 0.184 & 0.071 \\
\hline $\mathrm{S}_{4}(03$ December $)$ & 0.054 & 0.170 & 0.248 & 0.408 & 0.330 & 0.283 & 0.161 & 0.060 \\
\hline $\operatorname{LSD}(5 \%)$ & 0.013 & 0.015 & 0.053 & 0.055 & 0.039 & 0.029 & 0.016 & 0.009 \\
\hline
\end{tabular}

Based on the findings, at different sowing dates, CGR was higher in the early sown plants. The highest CGR was in $\mathrm{S}_{1}$ plants (Table 3). Similar result was obtained by Biswas et al. (2002) in blackgram and Hokmalipour et al. (2011) in canola. CGR increased up to a certain peak and thereafter declined and then increased gradually and sharply reached their highest value and again declined at the later stages of growth. Similar trend was also observed by Clarke and Simpson (1978) in rape and Mondal and Paul (1992) in mustard. In the present investigation, the highest NAR was found in the second sowing when the maximum temperature was 30C. Wilson (1966) reported that NAR of rape, sunflower and maize growse with temperature up to maximum at $28 \mathrm{C}$. With a few exceptions, NAR increased very slowly at the early stages, then reached its 
peak and declined thereafter (Table 4). A similar occurrence was evident in mustard by Mondal and Paul $(1992,1994)$, in rapeseed by Sarker and Paul (1993). S2 plants had the highest LAR at 1 ${ }^{\text {st }}$ harvest interval in the $1^{\text {st }}$ year and $S_{4}$ plants in the $2^{\text {nd }}$ year (Table 5$) . S_{1}$ plant had the lowest LAR at last harvest interval. Similar result was reported by Mondal and Paul $(1992,1994)$ in mustard. Lower LAR might be due to abscission of older leaves at the later growing stages. Similar result was also reported by Islam and Paul (1986) in rape, Mondal and Paul (1992) in mustard.

Table 3. Mean values of crop growth rate $\left(\mathrm{g} \mathrm{m}^{-2} \mathrm{day}^{-1}\right)$ at different growth stages as influenced by sowing date (mean of three replications) in two seasons.

\begin{tabular}{|c|c|c|c|c|c|c|c|}
\hline \multirow{2}{*}{ Sowing } & \multicolumn{7}{|c|}{ Days after sowing } \\
\hline & $21-28$ & $28-35$ & $35-42$ & $42-49$ & $49-56$ & $56-63$ & $63-70$ \\
\hline \multicolumn{8}{|c|}{$2006-2007$} \\
\hline $\mathrm{S}_{1}(18$ October) & 1.837 & 6.253 & 13.009 & 7.397 & 7.637 & 9.964 & 3.391 \\
\hline $\mathrm{S}_{2}$ (02 November) & 1.549 & 3.219 & 6.306 & 3.063 & 7.380 & 10.848 & 3.238 \\
\hline $\mathrm{S}_{3}(17$ November $)$ & 1.173 & 1.957 & 3.226 & 5.395 & 3.378 & 6.748 & 0.807 \\
\hline $\mathrm{S}_{4}(03$ December $)$ & 1.015 & 1.256 & 2.055 & 4.681 & 2.962 & 3.824 & 0.586 \\
\hline LSD(5\%) & 0.411 & 0.909 & 0.516 & 1.155 & 1.276 & 0.952 & 1.193 \\
\hline \multicolumn{8}{|c|}{ 2008-2009 } \\
\hline $\mathrm{S}_{1}(18$ October) & 1.411 & 3.953 & 10.628 & 10.557 & 7.281 & 10.024 & 7.353 \\
\hline $\mathrm{S}_{2}$ (02 November) & 1.030 & 3.087 & 6.010 & 4.114 & 5.736 & 9.572 & 2.732 \\
\hline $\mathrm{S}_{3}(17$ November $)$ & 0.974 & 1.917 & 2.300 & 5.993 & 3.195 & 5.989 & 0.770 \\
\hline $\mathrm{S}_{4}(03$ December $)$ & 0.798 & 1.367 & 1.908 & 4.523 & 3.054 & 3.058 & 0.146 \\
\hline LSD(5\%) & 0.099 & 0.549 & 0.925 & 0.715 & 1.013 & 0.743 & 0.819 \\
\hline
\end{tabular}

Table 4. Mean values of net assimilation rate $\left(\mathrm{g} \mathrm{cm}^{-2} \mathrm{day}^{-1}\right)$ at different growth stages as influenced by sowing date (mean of three replications) in two seasons.

\begin{tabular}{|c|c|c|c|c|c|c|c|}
\hline \multirow{2}{*}{ Sowing } & \multicolumn{7}{|c|}{ Days after sowing } \\
\hline & $21-28$ & $28-35$ & 3542 & $42-49$ & $49-56$ & $56-63$ & $63-70$ \\
\hline \multicolumn{8}{|c|}{ 2006-2007 } \\
\hline $\mathrm{S}_{1}(18$ October) & 0.0008 & 0.0009 & 0.0011 & 0.0007 & 0.0013 & 0.0026 & 0.0019 \\
\hline $\mathrm{S}_{2}$ (02 November) & 0.0007 & 0.0007 & 0.0008 & 0.0005 & 0.0019 & 0.0042 & 0.0024 \\
\hline $\mathrm{S}_{3}(17$ November $)$ & 0.0009 & 0.0006 & 0.0006 & 0.0012 & 0.0010 & 0.0027 & 0.0005 \\
\hline $\mathrm{S}_{4}(03$ December $)$ & 0.0010 & 0.0005 & 0.0006 & 0.0012 & 0.0008 & 0.0017 & 0.0004 \\
\hline LSD(5\%) & NS & NS & 0.00009 & 0.00009 & 0.0003 & 0.0003 & 0.001 \\
\hline \multicolumn{8}{|c|}{ 2008-2009 } \\
\hline $\mathrm{S}_{1}(18$ October $)$ & 0.0008 & 0.0009 & 0.0012 & 0.0011 & 0.0012 & 0.0027 & 0.0043 \\
\hline $\mathrm{S}_{2}$ (02 November) & 0.0006 & 0.0008 & 0.0009 & 0.0008 & 0.0014 & 0.0035 & 0.0021 \\
\hline $\mathrm{S}_{3}(17$ November $)$ & 0.0008 & 0.0007 & 0.0006 & 0.0017 & 0.0009 & 0.0025 & 0.0006 \\
\hline $\mathrm{S}_{4}(03$ December) & 0.0008 & 0.0006 & 0.0006 & 0.0012 & 0.0010 & 0.0015 & 0.0001 \\
\hline LSD(5\%) & 0.00009 & 0.00003 & 0.0003 & 0.0002 & 0.0003 & 0.0004 & 0.001 \\
\hline
\end{tabular}


Table 5. Mean values of leaf area ratio $\left(\mathrm{cm}^{2} \mathrm{~g}^{-1}\right)$ at different growth stages as influenced by sowing date (mean of three replications) in two seasons.

\begin{tabular}{|c|c|c|c|c|c|c|c|}
\hline \multirow{2}{*}{ Sowing } & \multicolumn{7}{|c|}{ Days after sowing } \\
\hline & $21-28$ & $28-35$ & $35-42$ & $42-49$ & $49-56$ & $56-63$ & $63-70$ \\
\hline \multicolumn{8}{|c|}{ 2006-2007 } \\
\hline $\mathrm{S}_{1}(18$ October) & 208.523 & 175.543 & 117.226 & 63.814 & 30.608 & 14.141 & 6.231 \\
\hline $\mathrm{S}_{2}$ (02 November) & 228.424 & 187.509 & 126.241 & 64.857 & 29.319 & 14.674 & 7.266 \\
\hline $\mathrm{S}_{3}(17$ November) & 197.746 & 182.292 & 140.227 & 73.353 & 36.053 & 19.461 & 8.954 \\
\hline $\mathrm{S}_{4}(03$ December) & 197.125 & 177.736 & 141.010 & 83.685 & 46.106 & 25.034 & 11.129 \\
\hline LSD(5\%) & NS & NS & NS & 9.38 & 4.29 & 1.20 & 0.74 \\
\hline \multicolumn{8}{|c|}{$2008-2009$} \\
\hline $\mathrm{S}_{1}(18$ October) & 201.974 & 171.649 & 123.997 & 65.918 & 32.015 & 14.732 & 5.558 \\
\hline $\mathrm{S}_{2}$ (02 November) & 222.018 & 189.214 & 127.307 & 63.871 & 32.346 & 16.897 & 6.696 \\
\hline $\mathrm{S}_{3}(17$ November) & 211.032 & 180.210 & 122.896 & 64.774 & 36.756 & 19.645 & 7.697 \\
\hline $\mathrm{S}_{4}(03$ December) & 224.312 & 171.809 & 139.051 & 83.937 & 42.669 & 22.971 & 9.434 \\
\hline LSD(5\%) & NS & NS & 8.43 & 5.32 & 2.63 & 0.95 & 0.72 \\
\hline
\end{tabular}

\section{Effect of weeding}

TDM was higher in all the weeding plants than in the control plants (Table 6). Increased weeding level increased TDM. $W_{2}$ treatment had the highest TDM. Similar trends of the effect of weeding were also observed in rapeseed by Rajput et al. (1993) and Rashid et al. (2007).

Table 6. Mean values of total dry matter $\left(\mathrm{gm}^{-2}\right)$ at different growth stages as influenced by weeding (mean of three replications) in two seasons.

\begin{tabular}{|c|c|c|c|c|c|c|c|c|}
\hline \multirow{2}{*}{ Weeding } & \multicolumn{8}{|c|}{ Days after sowing } \\
\hline & 21 & 28 & 35 & 42 & 49 & 56 & 63 & 70 \\
\hline & \multicolumn{8}{|c|}{ 2006-2007 } \\
\hline $\mathrm{W}_{0}$ (No weeding) & 4.274 & 10.533 & 23.972 & 52.161 & 82.376 & 111.988 & 157.910 & 174.119 \\
\hline $\mathrm{W}_{1}$ (One weeding) & 4.246 & 15.260 & 38.994 & 81.377 & 113.008 & 146.822 & 205.756 & 217.690 \\
\hline $\mathrm{W}_{2}$ (Two weeding) & 4.444 & 16.434 & 45.861 & 104.421 & 150.385 & 199.080 & 258.993 & 272.966 \\
\hline LSD(5\%) & NS & 0.36 & 1.15 & 1.78 & 2.23 & 2.33 & 1.88 & 2.96 \\
\hline \multicolumn{9}{|c|}{ 2008-2009 } \\
\hline $\mathrm{W}_{0}$ (No weeding) & 3.425 & 9.184 & 21.347 & 46.764 & 79.576 & 107.146 & 151.038 & 172.197 \\
\hline$W_{1}$ (One weeding) & 3.450 & 11.541 & 32.043 & 66.416 & 107.047 & 139.528 & 192.405 & 212.134 \\
\hline $\mathrm{W}_{2}$ (Two weeding) & 3.502 & 11.767 & 33.302 & 82.953 & 141.740 & 182.835 & 236.443 & 253.602 \\
\hline LSD(5\%) & NS & 0.24 & 1.13 & 1.95 & 2.81 & 1.36 & 1.95 & 2.19 \\
\hline
\end{tabular}

Higher LAI was in the weeding levels than in the control. The highest LAI was in $\mathrm{W}_{2}$ treatment. Miri and Rahimi (2009) reported that in rapeseed lack of weed competition and increased weed free duration can result in increasing crop LAl. Miri and Ghadiri (2006) also showed that in safflower plants weed free plots reached its maximum LAl earlier and maintained a maximum LAl for a longer period compared to weedy check (Table 7). Higher CGR was in the weeding plants than in the control (Table 8) and the highest CGR was in the $\mathrm{W}_{2}$ 
treatment. This result is in agreement with crops like rapeseed by Mousavi and Shimi (2004), in canola by Pourazar and Shimi (2004) and Marwat et al. (2005).

Table 7. Mean values of leaf area index at different growth stages as influenced by weeding (mean of three replications) in two seasons.

\begin{tabular}{|c|c|c|c|c|c|c|c|c|}
\hline \multirow{2}{*}{ Weeding } & \multicolumn{8}{|c|}{ Days after sowing } \\
\hline & 21 & 28 & 35 & 42 & 49 & 56 & 63 & 70 \\
\hline & \multicolumn{8}{|c|}{$2006-2007$} \\
\hline Wo(No weeding) & 0.089 & 0.217 & 0.405 & 0.577 & 0.372 & 0.277 & 0.162 & 0.053 \\
\hline $\mathrm{W}_{1}$ (One weeding) & 0.093 & 0.325 & 0.631 & 0.809 & 0.523 & 0.377 & 0.241 & 0.121 \\
\hline $\mathrm{W}_{2}$ (Two weeding) & 0.100 & 0.336 & 0.728 & 0.982 & 0.661 & 0.456 & 0.316 & 0.188 \\
\hline \multirow[t]{2}{*}{ LSD (5\%) } & NS & 0.031 & 0.060 & 0.076 & 0.053 & 0.032 & 0.017 & 0.013 \\
\hline & \multicolumn{8}{|c|}{ 2008-2009 } \\
\hline$W_{0}$ (No weeding) & 0.070 & 0.202 & 0.351 & 0.498 & 0.363 & 0.321 & 0.160 & 0.055 \\
\hline $\mathrm{W}_{1}$ (One weeding) & 0.075 & 0.254 & 0.518 & 0.694 & 0.465 & 0.357 & 0.215 & 0.100 \\
\hline $\mathrm{W}_{2}$ (Two weeding) & 0.076 & 0.246 & 0.546 & 0.848 & 0.611 & 0.464 & 0.265 & 0.106 \\
\hline LSD (5\%) & NS & 0.015 & 0.019 & 0.047 & 0.034 & 0.016 & 0.017 & 0.007 \\
\hline
\end{tabular}

Table 8. Mean values of crop growth rate $\left(\mathrm{g} \mathrm{m}^{-2} \mathrm{day}^{-1}\right)$ at different growth stages as influenced by weeding (mean of three replications) in two seasons.

\begin{tabular}{|l|c|c|c|c|c|c|c|}
\hline \multirow{2}{*}{ Weeding } & \multicolumn{7}{c|}{ Days after sowing } \\
\cline { 2 - 9 } & $21-28$ & $28-35$ & $35-42$ & $42-49$ & $49-56$ & $56-63$ & $63-70$ \\
\hline & \multicolumn{7}{|c|}{$2006-2007$} \\
\hline$W_{0}$ (No weeding) & 0.894 & 1.920 & 4.027 & 4.316 & 4.230 & 6.560 & 2.316 \\
\hline$W_{1}$ (One weeding) & 1.573 & 3.391 & 6.055 & 4.519 & 4.831 & 8.419 & 1.705 \\
\hline$W_{2}$ (Two weeding) & 1.713 & 4.204 & 8.366 & 6.566 & 6.956 & 8.559 & 1.996 \\
\hline LSD (5\%) & NS & 0.349 & 0.788 & 0.415 & 0.418 & 0.538 & NS \\
\hline & & & $2008-2009$ & & & \\
\hline$W_{0}$ (No weeding) & 0.823 & 1.738 & 3.631 & 4.687 & 3.939 & 6.270 & 3.023 \\
\hline$W_{1}$ (One weeding) & 1.156 & 2.929 & 4.910 & 5.804 & 4.640 & 7.554 & 2.818 \\
\hline$W_{2}$ (Two weeding) & 1.181 & 3.076 & 7.093 & 8.398 & 5.871 & 7.658 & 2.410 \\
\hline LSD (5\%) & 0.039 & 0.164 & 0.376 & 0.404 & 0.348 & 0.250 & 0.389 \\
\hline
\end{tabular}

The highest NAR was observed in Wo plants at 56-63 DAS (Table 9). NAR increased very slowly at the early stages, then reached its peak and declined thereafter. Similar result was reported by Yadav et al. (1995), Khan et al. (1995) and Singh et al. (2000) in rapeseed and mustard. The highest LAR was in the $\mathrm{W}_{1}$ plant at the first harvest interval and the lowest was in Wo plant at the last harvest interval (Table 10). 
Table 9. Mean values of net assimilation rate $\left(\mathrm{g} \mathrm{cm}^{-2} \mathrm{day}^{-1}\right)$ at different growth stages as influenced by weeding (mean of three replications) in two seasons.

\begin{tabular}{|l|c|c|c|c|c|c|c|}
\hline \multirow{2}{*}{ Weeding } & \multicolumn{7}{c|}{ Days after sowing } \\
\cline { 2 - 9 } & $21-28$ & $28-35$ & 3542 & $42-49$ & $49-56$ & $56-63$ & $63-70$ \\
\hline \multicolumn{7}{|c|}{$2006-2007$} \\
\hline$W_{0}$ (No weeding) & 0.0007 & 0.0006 & 0.0007 & 0.0010 & 0.0012 & 0.0032 & 0.0023 \\
\hline$W_{1}$ (One weeding) & 0.0009 & 0.0007 & 0.0007 & 0.0008 & 0.0011 & 0.0028 & 0.0009 \\
\hline$W_{2}$ (Two weeding) & 0.0009 & 0.0008 & 0.0009 & 0.0009 & 0.0014 & 0.0023 & 0.0008 \\
\hline LSD (5\%) & 0.00008 & 0.00007 & 0.00009 & 0.00008 & 0.00009 & 0.0002 & 0.001 \\
\hline & & $2008-2009$ & & & 0.0027 & 0.0026 \\
\hline$W_{0}$ (No weeding) & 0.0007 & 0.0006 & 0.0008 & 0.0012 & 0.0011 & 0.0027 \\
\hline$W_{1}$ (One weeding) & 0.0008 & 0.0008 & 0.0007 & 0.0011 & 0.0012 & 0.0027 & 0.0016 \\
\hline$W_{2}$ (Two weeding) & 0.0008 & 0.0008 & 0.0010 & 0.0012 & 0.0011 & 0.0023 & 0.0012 \\
\hline LSD (5\%) & 0.00006 & 0.00005 & 0.0002 & NS & Ns & 0.0003 & 0.0003 \\
\hline
\end{tabular}

Table 10. Mean values of leaf area ratio $\left(\mathrm{cm}^{2} \mathrm{~g}^{-1}\right)$ at different growth stages as influenced by weeding (mean of three replications) in two seasons.

\begin{tabular}{|l|c|c|c|c|c|c|c|}
\hline \multirow{2}{*}{ Weeding } & \multicolumn{7}{c|}{ Days after sowing } \\
\cline { 2 - 9 } & $21-28$ & $28-35$ & $35-42$ & $42-49$ & $49-56$ & $56-63$ & $63-70$ \\
\hline \multicolumn{7}{|c|}{$2006-2007$} \\
\hline W $_{\text {(No weeding) }}$ & 204.518 & 182.900 & 137.223 & 74.563 & 35.882 & 16.985 & 6.135 \\
\hline W $_{1}$ (One weeding) & 212.452 & 182.894 & 130.855 & 72.332 & 36.832 & 19.198 & 8.845 \\
\hline W $_{2}$ (Two weeding) & 206.894 & 176.516 & 125.450 & 67.386 & 33.850 & 18.799 & 10.205 \\
\hline LSD (5\%) & NS & NS & 6.60 & 4.18 & 1.79 & 0.87 & 0.64 \\
\hline & \multicolumn{7}{|c|}{$2008-2009$} \\
\hline W $_{0}$ (No weeding) & 212.377 & 185.309 & 129.087 & 70.758 & 38.900 & 19.010 & 6.417 \\
\hline W $_{1}$ (One weeding) & 220.772 & 177.231 & 128.975 & 69.773 & 34.475 & 18.391 & 8.195 \\
\hline$W_{2}$ (Two weeding) & 211.353 & 172.121 & 126.877 & 68.344 & 34.465 & 18.282 & 7.427 \\
\hline LSD (5\%) & NS & 8.14 & NS & NS & 1.61 & NS & 0.49 \\
\hline
\end{tabular}

\section{Conclusion}

TDM, LAI, CGR decreased with late sowing. The first sowing plants had higher values of the above characters. There was no clear pattern of LAR. The highest and lowest TDM, LAI and CGR were found in the $W_{2}$ and $W_{0}$ treatments, respectively. $W_{1}$ treatment had the highest NAR and LAR and the lowest were found in $W_{0}$ treatment. 


\section{References}

Bangladesh bureau of statistics planning division, ministry of planning government of the people`s republic of Bangladesh Dhaka, Bangladesh. www. bbs. gov. bd

Clarke JM, Simpson GM. 1978. Growth analysis of Brassica napus cv. Tower. Can J Plant Sci 58, 587-595. http://dx.doi.org/10.4141/cjps78-092

Dehdeshti SM, Solimani A, Majdenasiri B. 2006. Effect of delayed planting on physiological indicators of rapeseed cultivars Brassica napus. Journal of Research in Agricultural Sciences. 4, 2.

Ghosh K, Maiti S, Mondal NN, Pal S. 2003. Effect of dates of sowing and sources of sulphur on growth and yield of sesame. Bangladesh J Agril Res 28(4),465-474.

Gomez KA, Gomez AA. 1984. Statistical Procedure for Agricultural Research Intl. Rice Res. Inst. John Wiley, New York. $139 \mathrm{pp}$.

Hokmalipour S, Tobe A, Jafarzadeh B, Darbandi MH. 2011. Effect of sowing date on physiological growth indices in canola (Brassica napus L.) cultivars in meshkin region. World Applied Sciences Journal 14(4), 514-522.

Hussain A, Nawaz M, Azam M, Kamran M. 1997. Growth analysis of chickpea (Cicer arietinum L.) sown at different sowing dates and populations. J Agric Res 35 (4), 227-236.

Islam MM, Paul NK. 1986. Comparative growth analysis of six cultivars of rape seed (Brassica campestris L.). Bangladesh J Agril Sci 13, 35-39.

Joel DM, Kleifeld Y, Goshen DL. 1995. Transgenic crops against parasites. Nature London 374, 220-221. http://dx.doi.org/10.1038/374220a0

Khan RU, Khan NA, Ahmad M. 1995. Performance of Treflan: a preplant applied herbicide in rapeseed and mustard. Sarh J Agric 11, 647-655.

Kundu PB,Paul NK. 1998. Effect of soil moisture on growth and yield of rape (Brassica campestris L.). Rajshahi University Studies. Part-B. 26, 119-128.

Marwat KB, Saeed M, Gul B, Hussain Z, Khan NI. 2005. Efficiency of different pre and post-emergence herbicides for weed management in canola in higher altitudes. Pakistan J Weed Sci Res 11, 75-80.

Miri HR, Ghadiri H. 2006. Determination of the critical period of weed control in fall-grown safflower (Carthamus tinctorius). Iran J Weed Sci 2, 1-16.

Miri HR, Rahimi Y. 2009. Effects of combined and separate herbicide application on rapeseed and its weeds in Southern Iran. Int J. Agric Biol 11, 257-260.

Mondal RK, Paul NK. 1992. Growth and physiological characters of mustard under rainfed and irrigated conditions. Bangladesh J Agril Res 17, 29-36.

Mondal RK, Paul NK. 1994. Growth analysis using classical and functional methods in relation to soil moisture in mustard (Brassica juncea L.) J Agron \& Crop Sci 173, 230-240. http://dx.doi.org/10.1111/j.1439-037X.1994.tb00559.x

Mousavi SK, Shimi P. 2004. Efficiency evaluation of low rate haloxyfob-R methyl as compared with other graminicides common in oilseed rape field. Proc. 16th Iranian Plant Protection Cong. 28 Agust-1 September 2004. Tabriz University, Iran.

Paul NK, Sarker DK. 2003. Grain yield and its components of wheat (Triticum aestivam L.) as influenced by sowing date. Rajshahi Univ. Studies. Pant B. J. 31:169-176.

Paul NK, Kundu PB. 1991. Influence of soil moisture on growth, water use and yield of rapeseed (Brassica campestris L.). Proc Int Conf PI Physiol Banaras Hindu Univ. India. pp. 35-42.

Pourazar R ,Shimi P. 2004. Evaluating efficiency of Metazachlor in Canola (Brassica napus). Proc. 16th Iranian Plant Protection Cong. 28 Agust-1 September 2004. Tabriz University, Iran.

Prodhan AC, Ghose DC. 1986. Growth and yield variations of finger millet varieties due to nitrogen fertilization. Indian J Agron 31, 63-68. 
Rajput RL, Gautam DS, Verma OP. 1993. Studies on cultural and chemical weed control in mustard (Brassica juncea). Gujarat Agric Univ Res J. 8(2), 1-5.

Rashid R, Karim MF, M Hasanuzzaman. 2007. Response of rapeseed (Brassica campestris L.) to different nitrogen doses and number of weeding. Middle-East J Sci Res 2(3-4), 146-150.

Samanta SC, Gaffer MA, Kamal AMA, Samad MA. 1997. Growth and yield of prosomillet cultivars as influenced by sowing date. Bangladesh J Agril Sci 24, 37-41.

Sarker MA, Paul NK. 1993. Physiological analysis of delayed sowing on yield of rapeseed (Brassica campestris L..). Bangladesh J Agric 18, 25-33.

Singh SK, Jain NK, Poonia BL. 2000. Integrated weed management in Indian mustard (Brassica juncea). Indian J Agric Sci 70, 850-852.

Wilson JW. 1966. Effect of temperature on net assimilation rate. Ann Bot 30, 753-760.

Yadav RP, Shrivastava UK, Yadav KS. 1995. Yield and economic analysis of weed control practices in Indian mustard (Brassica juncea). Indian J Agron 40, 122-124. 